\title{
Vitamin D3 protects articular cartilage by inhibiting the Wnt/ $\beta$-catenin signaling pathway
}

\author{
TAO YANG $^{1 *}$, WEI SUN ${ }^{2,3^{*}}$, YUAN-HUI DUAN ${ }^{1}$, YUN-BO SUN $^{1}$, \\ YI-MING REN $^{1}$, WEI-YU HOU ${ }^{1}$ and MENG-QIANG TIAN ${ }^{1}$ \\ ${ }^{1}$ Department of Joint and Sports Medicine, Tianjin Union Medical Center, Tianjin 300121; \\ ${ }^{2}$ The Postdoctoral Research Station, Chinese Academy of Medical Sciences and \\ Peking Union Medical College, Beijing 100730; ${ }^{3}$ Department of Respiratory, \\ Affiliated Zhongshan Hospital of Dalian University, Dalian, Liaoning 116001, P.R. China
}

Received September 15, 2019; Accepted April 1, 2020

DOI: $10.3892 /$ etm.2020.8839

\begin{abstract}
Low expression levels of 25-hydroxyvitamin D (vitamin D3) in the blood have been reported to be associated with the progression of osteoarthritis; however, the mechanisms by which this occurs remain unclear. The present study aimed to determine the effects of vitamin D3 on chondrocytes. MTT assays were used to determine whether vitamin D3 affects chondrocytes viability. Primary chondrocytes were treated with control culture medium, vitamin D3, tumor necrosis factor (TNF)- $\alpha$, TNF- $\alpha+$ PNU-74654 [Wingless-related integration site (Wnt)/ $\beta$-catenin signaling pathway inhibitor] or TNF- $\alpha+$ vitamin D3. Reverse transcription-quantitative PCR and western blotting were utilized to measure the gene and protein expression of collagen II, aggrecan, matrix metalloproteinase (MMP)-3 and MMP-13, A disintegrin and metalloproteinase with thrombospondin motifs (ADAMTS)-4, ADAMTS-5, Wnt-3a and nuclear $\beta$-catenin. The results demonstrated that TNF- $\alpha$ reduced the expression levels of aggrecan and collagen II, and increased the expression levels of MMP-3, MMP-13, ADAMTS-4 and ADAMTS-5. Furthermore, vitamin D3 and PNU-74654 were observed to partially attenuate the effects induced by TNF- $\alpha$. Moreover, similar findings were reported following co-treatment with vitamin D3 and TNF- $\alpha$. Western blotting data revealed that TNF- $\alpha$ increased Wnt-3a and $\beta$-catenin protein levels in chondrocytes, while Vitamin D3 and PNU-74654 decreased the expression levels of Wnt-3a and nuclear $\beta$-catenin. In conclusion, the findings of the present study provided
\end{abstract}

Correspondence to: Dr Meng-Qiang Tian, Department of Joint and Sports Medicine, Tianjin Union Medical Center, 190 Jieyuan Road, Tianjin 300121, P.R. China

E-mail: mengqiang_tian@126.com

${ }^{*}$ Contributed equally

Key words: vitamin D3, Wnt/ $\beta$-catenin, osteoarthritis, cartilage, chondrocyte evidence to suggest that vitamin D3 may prevent articular cartilage degeneration and osteoarthritic disease progression by inhibiting the expression levels of MMP-3, MMP-13, ADAMTS- 4 and ADAMTS-5 through suppressing the Wnt/ $\beta$-catenin signaling pathway. These results suggested that vitamin D3 may be of therapeutic value for the prevention and treatment of osteoarthritis.

\section{Introduction}

Osteoarthritis of the knee is a common degenerative joint condition (1). According to the Global Burden of Disease study, the global age-standardized prevalence of knee OA is approximately $3.8 \%$ from 1990 to 2010 (1). The primary manifestations of osteoarthritis include the destruction of the cartilage and sclerosis of the subchondral bone (2). However, although osteoarthritis is the most common joint disease, the specific biological mechanisms underlying its pathogenesis remain poorly understood (3).

Low expression levels of blood 25-hydroxyvitamin D (vitamin D) have been revealed to be associated with the progression of osteoarthritis, whereby vitamin $\mathrm{D}$ has been discovered to protect against osteoarthritis (4). The vitamin D receptor is expressed on the surface of chondrocytes, providing a basis for vitamin D action on articular chondrocytes (5). However, the specific mechanism through which vitamin D protects articular chondrocytes from osteoarthritis remains unclear.

The Wingless-related integration site (Wnt) signaling pathway component, $\beta$-catenin, stimulates bone hypertrophy, matrix mineralization and matrix metalloproteinase (MMP)-13 expression; the overexpression of $\beta$-catenin in chondrocytes was demonstrated to strongly induce the expression of matrix degrading enzymes (6). In pathological conditions, the $\mathrm{Wnt} / \beta$-catenin signaling pathway has been indicated to activate cartilage matrix catabolism and destroy articular cartilage (7).

The binding of vitamin $\mathrm{D}$ to the vitamin $\mathrm{D}$ receptor was discovered to inhibit the Wnt/ $\beta$-catenin signaling pathway (8). The subsequent binding to nuclear $\beta$-catenin promotes the translocation of $\beta$-catenin from the nucleus, where it binds 
to an oligomeric casein kinase/adenomatous polyposis coli/glycogen synthase kinase 3/ $\beta$-axis complex, which mediates $\beta$-catenin phosphorylation and accelerates $\beta$-catenin hydrolysis (9). These events lead to the reduction in $\beta$-catenin levels and the inhibition of the Wnt signaling pathway (9). This may be one of the mechanisms by which vitamin $D$ protects articular cartilage. Thus, the present study aimed to investigate whether vitamin $\mathrm{D}$ affected chondrocyte fate through modulating the Wnt/ $\beta$-catenin signaling pathway.

\section{Materials and methods}

Chondrocyte isolation and culture. The present study was approved by the Animal Care and Use Committee of Tianjin Union Medical Center and Affiliated Zhongshan Hospital of Dalian University. A total of 10 female Sprague Dawley Rats (age, 4 weeks; weight, $80 \mathrm{~g}$ ) were obtained from Charles River Laboratories, Inc. The rats were kept in a clean-grade animal house at a temperature of $20 \pm 2^{\circ} \mathrm{C}$, a humidity of $60 \pm 5 \%$, with $12 \mathrm{~h} \mathrm{light/dark}$ cycles and free access to food and water. Following anesthesia with pentobarbital sodium $(30 \mathrm{mg} / \mathrm{kg}$; Shanghai Ziyuan Pharmaceutical Co., Ltd.), the articular cartilage from the knee joint and femoral head tissue was removed and washed three times with PBS, minced into small pieces and digested with $0.2 \%$ type II collagenase (Gibco; Thermo Fisher Scientific, Inc.) in DMEM/F12 (Hyclone; GE Healthcare Life Sciences), supplemented with $100 \mathrm{U} / 1$ penicillin and $100 \mu \mathrm{g} / \mathrm{ml}$ streptomycin (Gibco; Thermo Fisher Scientific, Inc.) at $37^{\circ} \mathrm{C}$ for $6-8 \mathrm{~h}$. The cell suspension was centrifuged at $200 \mathrm{x} \mathrm{g}$ for $10 \mathrm{~min}$ at room temperature. Harvested cells were subsequently cultured in DMEM/F12, supplemented with $10 \%$ FBS (HyClone; Cytiva) at $37^{\circ} \mathrm{C}$ and $5 \% \mathrm{CO}_{2}$. The isolated cells were used for the following experiments.

MTT assay. The cytotoxicity of vitamin D3 on rat chondrocytes was determined using MTT assays. Briefly, $1 \times 10^{4}$ chondrocytes/well were cultured in 96-well plates with $10 \mathrm{nM}$ vitamin D3 (Beijing Solarbio Science \& Technology Co., Ltd.) for $72 \mathrm{~h}$ at $37^{\circ} \mathrm{C}$. MTT assays were performed according to the manufacturer's protocol. Briefly, $20 \mu \mathrm{l}$ MTT reagent $(5 \mathrm{mg} / \mathrm{ml}$; Beijing Solarbio Science and Technology Co., Ltd.) was added to each well and the plate was incubated at $37^{\circ} \mathrm{C}$ for $4 \mathrm{~h}$. The medium was removed and $150 \mu 1$ DMSO was subsequently added into each well. The absorbance at $570 \mathrm{~nm}$ was measured using a microplate reader (Bio-Rad Laboratories, Inc.). A single well contained chondrocytes without vitamin D3 treatment (treatment control) and another well did not contain cells (blank control).

In vitro cell treatment. Following 4-5 days in culture, the confluent monolayers of chondrocytes in the culture plates were washed three times with DMEM/F12, supplemented with 10\% FBS (Hyclone; Cytiva) and incubated overnight with serum-free DMEM at $37^{\circ} \mathrm{C}$. Serum-starved chondrocytes were left untreated (control), treated with vitamin D3 $(10 \mathrm{nM})$, treated with tumor necrosis factor (TNF)- $\alpha(10$ $\mathrm{ng} / \mathrm{ml}$; PeproTech, Inc.), treated with both TNF- $\alpha$ and PNU-74654 (10 $\mu \mathrm{M}$; Selleck Chemicals) or treated with both TNF- $\alpha(10 \mathrm{ng} / \mathrm{ml})$ and vitamin D3 (10 nM). Following $48 \mathrm{~h}$ of incubation at $37^{\circ} \mathrm{C}$ with the respective treatments, the chondrocytes were harvested for RNA and protein isolation using reverse transcription-quantitative PCR (RT-qPCR) and western blotting analysis, respectively.

$R T-q P C R$. RT-qPCR was performed according to a previously described method (10). Briefly, a confluent monolayer of rat chondrocytes was washed three times with PBS and total RNA was extracted using TRIzol ${ }^{\circledR}$ reagent (Invitrogen; Thermo Fisher Scientific, Inc.), according to the manufacturer's protocol. Total RNA was reverse transcribed into cDNA using a PrimeScript RT reagent kit (Takara Bio, Inc.). The thermocycling conditions were as follows: $37^{\circ} \mathrm{C}$ for $15 \mathrm{~min}$ and $85^{\circ} \mathrm{C}$ for $5 \mathrm{sec}$. RT-qPCR was performed using a TB Green ${ }^{\circledR}$ Premix Ex Taq ${ }^{\mathrm{TM}}$ II kit (Takara Bio, Inc.), according to the manufacturer's protocols, on a StepOnePlus Real-Time PCR System (Applied Biosystems; Thermo Fisher Scientific, Inc.). The thermocycling conditions were as follows: Initial denaturation at $95^{\circ} \mathrm{C}$ for $30 \mathrm{sec}$ (stage 1), followed by 40 cycles of $95^{\circ} \mathrm{C}$ for $5 \mathrm{sec}$ and $60^{\circ} \mathrm{C}$ for $30 \mathrm{sec}$ (stage 2). The primers pairs used to the qPCR was presented in Table I. Expression levels were quantified using the $2^{-\Delta \Delta C a}$ method (11) and normalized to GAPDH expression. All experiments were carried out in triplicate.

Western blotting. Rat chondrocytes were washed with ice-cold PBS and total protein was extracted using RIPA lysis buffer (Beyotime Institute of Biotechnology) for $20 \mathrm{~min}$ on ice. The cell lysates were scraped into $1.5 \mathrm{ml}$ microcentrifuge tubes using cell scrapers and centrifuged for $10 \mathrm{~min}$ at $12,000 \mathrm{x} \mathrm{g}$ at $4^{\circ} \mathrm{C}$. The supernatants were collected as the cytoplasmic extract, whilst the pellets were resuspended in RIPA lysate buffer (Beyotime Institute of Biotechnology) and kept on ice for $30 \mathrm{~min}$. The cell lysates were subsequently centrifuged for $10 \mathrm{~min}$ at $12,000 \mathrm{x} \mathrm{g}$ at $4^{\circ} \mathrm{C}$ and the supernatants were collected as nuclear extracts. Total protein in each sample was quantified using the bicinchoninic acid (BCA) assay method using an enhanced BCA Protein Assay kit (Beyotime Institute of Technology). The protein samples were denatured at $95^{\circ} \mathrm{C}$ for $5 \mathrm{~min}$ and $30 \mu \mathrm{g}$ protein/lane was separated by $10 \%$ SDS-PAGE. The separated proteins were transferred onto a PVDF membrane (EMD Millipore) and incubated for $1 \mathrm{~h}$ with 5\% non-fat milk at room temperature. The membranes were washed with TBS-tween (TBST, $0.5 \%$ Tween) and incubated with the following primary antibodies at $4^{\circ} \mathrm{C}$ overnight, The antibodies used were as follows: Anti-aggrecan (1:1,000; cat. no. 13880-1-AP; Wuhan Sanying Biotechnology), anti-collagen II (1:500; cat. no. 15943-1-AP; Wuhan Sanying Biotechnology), anti-MMP-3 (1:1,000; cat. no. 17873-1-AP; Wuhan Sanying Biotechnology), anti-MMP-13 (1:1000, cat. no. 18165-1-AP, Wuhan Sanying Biotechnology), anti-A disintegrin and metalloproteinase with thrombospondin motifs (ADAMTS)-4 (1:1,000; cat. no. bs-4191R; BIOSS), anti-ADAMTS-5 (1:1,000; cat. no. bs-3573R; BIOSS), anti- $\beta$-catenin (1:1,000; cat. no. 17565-1-AP; Wuhan Sanying Biotechnology), anti-Wnt-3a (1:1,000; cat. no. bs-23277R; BIOSS), anti- $\beta$-actin (1:1,000; cat. no. 20536-1-AP; Wuhan Sanying Biotechnology) and anti-Lamin B1 (1:1,000; cat. no. 12987-1-AP; Wuhan Sanying Biotechnology). Following the primary antibody incubation, the membranes were washed with TBST and incubated with a horseradish peroxidase-conju- 
Table I. Primers used for reverse transcription-quantitative PCR.

\begin{tabular}{ll} 
Gene & \multicolumn{1}{c}{ Primer sequence (5'-3') } \\
\hline GAPDH & F: GGAATCCACTGGCGTCTTCA \\
& R: GGTTCACGCCCATCACAAAC \\
Aggrecan & F: TAAACCCGGTGTGAGAACCG \\
& R: CCTGGGTGACAATCCAGTCC \\
Collagen II & F: CCCCTGCAGTACATGCGG \\
& R: CTCGACGTCATGCTGTCTCAAG \\
MMP-3 & F: GCATTGGCTGAGTGAAAGAGAC \\
& R: ATGATGAACGATGGACAGATGA \\
MMP-13 & F: CAGTTGACAGGCTCCGAGAA \\
& R: CGTGTGCCAGAAGACCAGAA \\
ADAMTS-4 & F: GCCAGCAACCGAGGTCCCATA \\
& R: CCACCACCAGTGTCTCCACGAAT \\
ADAMTS-5 & F: GACAAGAGTCTGGAJGGTGAGCAA \\
& R: GCTGCATCGTAGTGCTCCTCAT
\end{tabular}

MMP, matrix metalloproteinase; ADAMTS, A disintegrin and metalloproteinase with thrombospondin motifs; F, forward; R, reverse.

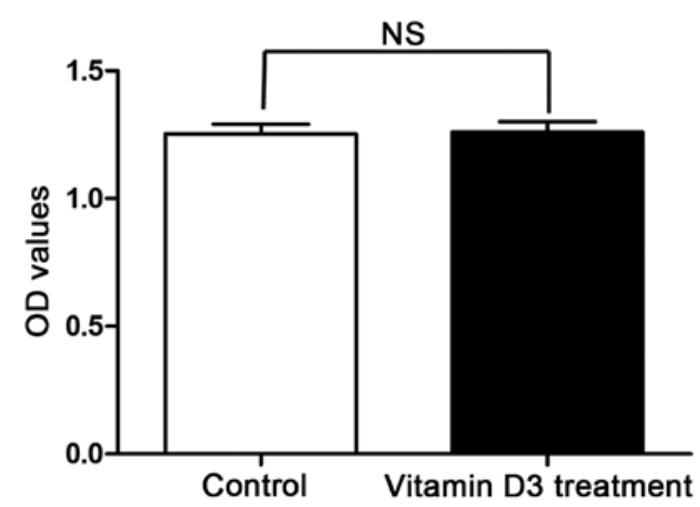

Figure 1. MTT assay was used to demonstrate that vitamin D3 had no significant effect on chondrocyte viability following $72 \mathrm{~h}$ treatment. NS, non-significant; OD, optical density.

gated goat anti-rabbit IgG secondary antibody (1:5,000; cat. no. SA00001-2; Wuhan Sanying Biotechnology) at room temperature for $1 \mathrm{~h}$. Protein bands were visualized using an enhanced chemiluminescence (Thermo Fisher Scientific, Inc.) with a ChemiDoc XRS+ (Bio-Rad Laboratories, Inc.) and quantified by Image Plus pro software (version 6.0; Media Cybernetics, Inc.).

Statistical analysis. All experiments were performed in triplicate. Statistical analysis was performed using GraphPad Prism software (version 8.02; GraphPad Software, Inc.) and data are presented as the mean \pm standard deviation. Statistical differences between two groups were determined using a Student's t-test, whereas statistical differences between multiple groups were performed using a one-way ANOVA, followed by a Tukey's test or a Tamhane's T2 test for data with a homogenous or non-homogenous variance, respectively.
$\mathrm{P}<0.05$ was considered to indicate a statistically significant difference.

\section{Results}

Viability of chondrocytes following vitamin D3 treatment. The MTT assay results demonstrated that there was no significant difference in the viability of chondrocytes treated with vitamin D3 compared with the control chondrocytes, indicating that there was no significant cytotoxic effect of treating rat chondrocytes with $10 \mathrm{nM}$ vitamin D3 for $72 \mathrm{~h}$ (Fig. 1).

Effects of vitamin D3 treatment on aggrecan and COL2A1 expression levels in rat chondrocytes. Articular cartilage is mainly composed of collagen II and proteoglycan, of which aggregated proteoglycan is the most abundant (12). In the present study, RT-qPCR was used to determine the expression levels of collagen II and the proteoglycan, aggrecan. The mRNA expression levels of gene encoding aggrecan (ACAN) and gene encoding collagen II (COL2A1) following TNF- $\alpha$ treatment were significantly reduced (Fig. 2A and B) compared with negative controls. However, the combined treatment with the Wnt/ $\beta$-catenin signaling pathway inhibitor PNU-74654 and TNF- $\alpha$ significantly increased the ACAN and COL2A1 mRNA expression levels in the chondrocytes compared with the chondrocytes in the TNF- $\alpha$ group. Similarly, the combined treatment of vitamin D3 and TNF- $\alpha$ also significantly increased ACAN and COL2A1 mRNA expression levels in the chondrocytes compared with TNF- $\alpha$ treatment alone. However, combined treatment of vitamin D3 and TNF- $\alpha$ exhibited lower ACAN and COL2A1 gene expression compared with co-treatment of PNU-74654 and TNF- $\alpha$ (Fig. 2A and B). Western blotting analysis also revealed that the protein expression levels of aggrecan and COL2A1 followed the same trend as the RT-qPCR results (Fig. 2C).

Effects of vitamin D3 treatment on TNF- $\alpha$-induced rat chondrocytes. To determine the regulatory effects of vitamin D3 on MMP-3 and MMP-13 expression levels, the activation of these two metalloproteinases by vitamin D3 in rat chondrocytes was investigated. TNF- $\alpha$ treatment significantly increased the expression levels of MMP-3 and MMP-13 mRNA in rat chondrocytes compared with the control and vitamin D3 group (Fig. 3A and B). Notably, co-treatment of PNU-74654 and TNF- $\alpha$ significantly inhibited the TNF- $\alpha$-induced increases in MMP-3 and MMP-13 expression levels. In addition, the co-treatment of vitamin D3 and TNF- $\alpha$ significantly reduced the increased expression levels of MMP-3 and MMP-13 induced by TNF- $\alpha$ (Fig. 3A and B). Furthermore, vitamin D3 and TNF- $\alpha$ treatment group had higher MMP-3 and MMP-13 gene expression levels compared with the PNU-74654 and TNF- $\alpha$ treatment group. The western blot data for MMP-3 and MMP-13 protein expression levels revealed similar results to the RT-qPCR analysis (Fig. 3E). The expression levels of ADAMTS-4 and ADAMTS-5 were also significantly increased in chondrocytes treated with TNF- $\alpha(10 \mathrm{ng} / \mathrm{ml})$ compared with the chondrocytes in the control and vitamin D3 treatment groups (Fig. 3C and D). However, the TNF- $\alpha$-induced increases in ADAMTS-4 and ADAMTS-5 expression levels were effectively attenuated by the co-treatment with both 

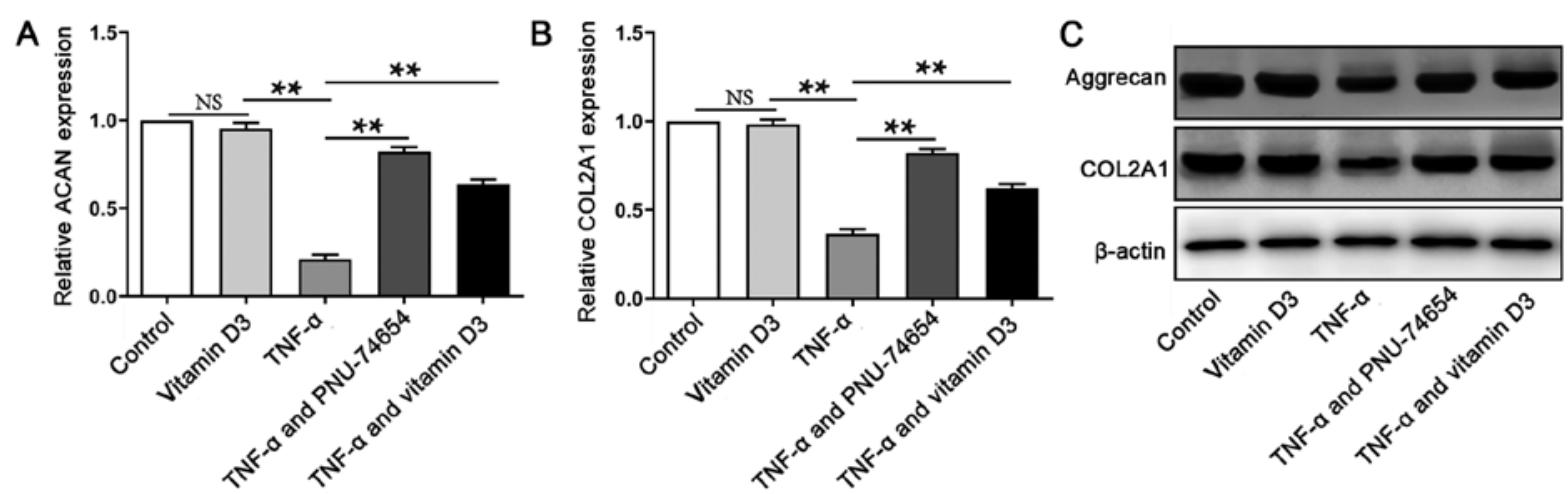

Figure 2. Vitamin D3 treatment inhibits TNF- $\alpha$-induced damage in chondrocytes. Reverse transcription-quantitative PCR was used to investigate the expression levels of (A) ACAN and (B) COL2A1 in chondrocytes. TNF- $\alpha$ treatment significantly reduced the mRNA expression levels of ACAN and COL2A1 in chondrocytes, while vitamin D3 and the $\beta$-catenin inhibitor (PNU-74654) significantly increased the expression of ACAN and COL2A1 in chondrocytes treated with TNF- $\alpha$. (C) Western blotting was used to investigate the expression levels of aggrecan and COL2A1. TNF- $\alpha$ treatment reduced the aggrecan and COL2A1 expression levels at the protein level, while vitamin D3 and the $\beta$-catenin inhibitor (PNU-74654) significantly counteracted this effect. ${ }^{* *} \mathrm{P}<0.01$. TNF- $\alpha$, tumor necrosis factor- $\alpha$; ACAN, aggrecan; COL2A1, collagen II; NS, non-significant.
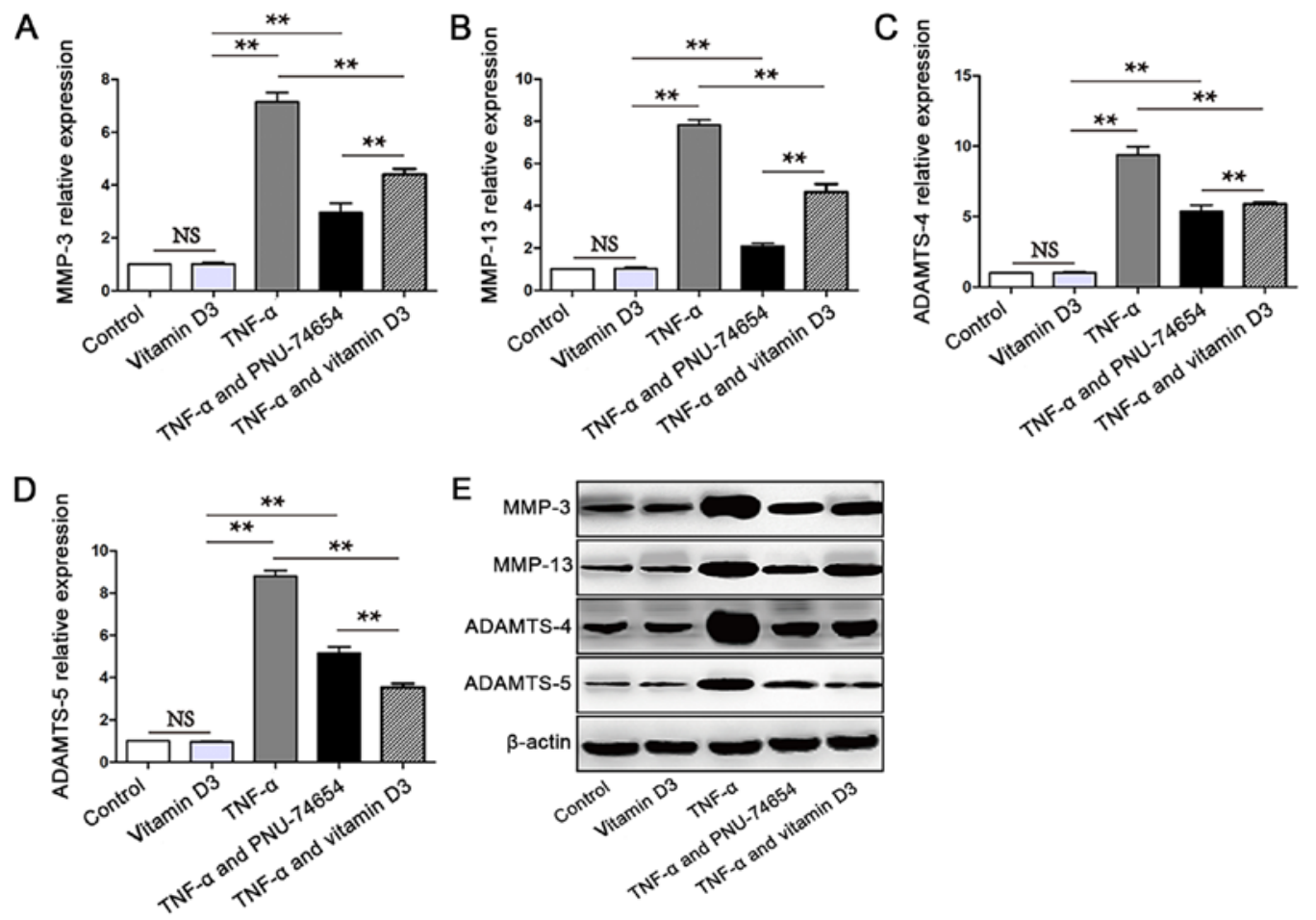

Figure 3. Vitamin D3 treatment attenuates the increased expression levels of MMP-3, MMP-13, ADAMTS-4 and ADAMTS-5 induced by TNF- $\alpha$. Reverse transcription-quantitative PCR was used to analyze the expression levels of (A) MMP-3, (B) MMP-13, (C) ADAMTS-4 and (D) ADAMTS-5. TNF- $\alpha$ treatment significantly increased the mRNA expression levels of MMP-3, MMP-13, ADAMTS-4 and ADAMTS-5, while vitamin D3 and PNU-74654 co-treatment significantly inhibited the expression levels of these mRNAs in chondrocytes induced with TNF- $\alpha$. (E) Western blotting was used to determine the protein expression levels of MMP-3, MMP-13, ADAMTS-4 and ADAMTS-5. TNF- $\alpha$ treatment increased MMP-3, MMP-13, ADAMTS-4 and ADAMTS-5 expression at the protein level. Vitamin D3 and PNU-74654 inhibited the expression of MMP-3, MMP-13, ADAMTS-4 and ADAMTS-5 induced by TNF- $\alpha$ at the protein level. ${ }^{* *} \mathrm{P}<0.01$. MMP, matrix metalloproteinase; ADAMTS, A disintegrin and metalloproteinase with thrombospondin motifs; TNF- $\alpha$, tumor necrosis factor- $\alpha$; NS, non-significant.

PNU-74654 or vitamin D3. Similarly, vitamin D3 and TNF- $\alpha$ treatment group had higher ADAMTS-4 and ADAMTS-5 gene expression level than PNU-74654 and TNF- $\alpha$ treatment group (Fig. 3C-E).

Effects of vitamin D3 treatment on the Wnt/ $\beta$-catenin signaling pathway. TNF- $\alpha$ treatment increased Wnt- $3 \mathrm{a}$ and nuclear $\beta$-catenin expression levels compared with control or vitamin D3 group, which suggested that the Wnt/ $\beta$-catenin signaling pathway was activated (Fig. 4A and B). Conversely, the co-treatment with vitamin D3 or PNU-74654 revealed a reduction in both Wnt-3a and nuclear $\beta$-catenin expression levels. And, vitamin D3 and TNF- $\alpha$ treatment group have weaker inhibitory effect on Wnt-3a and nuclear $\beta$-catenin than PNU-74654 and TNF- $\alpha$ treatment group. (Fig. 4A and B). 

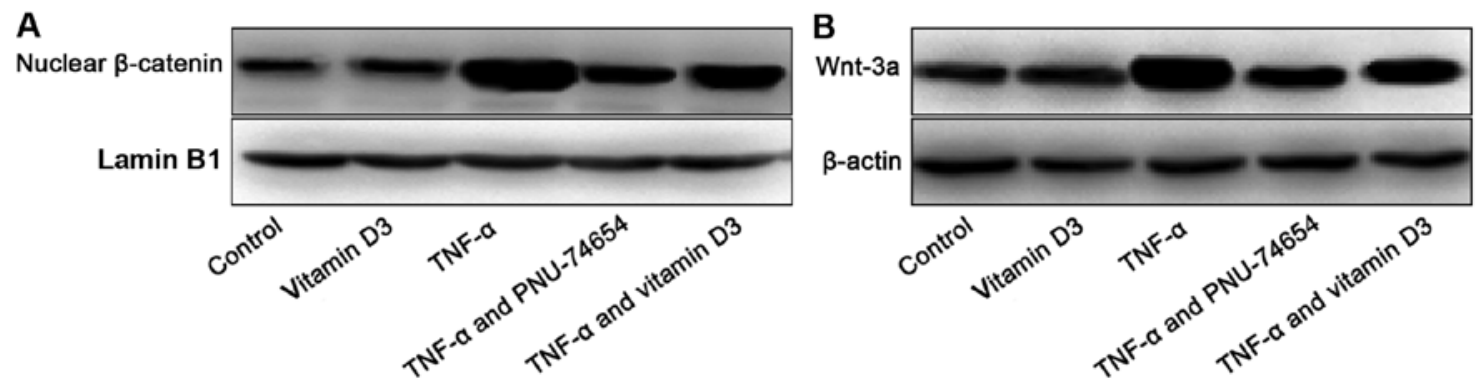

Figure 4. Vitamin D reversed the increased expression of Wnt-3a and nuclear $\beta$-catenin protein caused by TNF- $\alpha$. Western blotting was used to determine the expression levels of (A) nuclear $\beta$-catenin and (B) Wnt-3a. TNF- $\alpha$ treatment increased the expression levels of Wnt-3a and nuclear $\beta$-catenin, while vitamin D3 and PNU-74654 treatment inhibited the TNF- $\alpha$-induced increases in the expression of Wnt-3a and nuclear $\beta$-catenin. Wnt, Wingless-related integration site; TNF- $\alpha$, tumor necrosis factor- $\alpha$.

\section{Discussion}

It was previously established that vitamin D3 delayed the progression of knee osteoarthritis, although there is controversy surrounding the specific mechanisms involved (13). The characteristic gradual decomposition of extracellular matrix (ECM) in OA and the subsequent degradation of articular cartilage (14) have been demonstrated to be mediated by interleukin-1 $\beta$ (15), TNF- $\alpha$ (16) and other factors (17).

Articular cartilage is a special type of connective tissue; the highly organized ECM consists of major macromolecules, including the proteoglycan aggrecan, the most abundant proteoglycan found in the cartilage and COL2A1 (18). In the present study, TNF- $\alpha$ treatment decreased the levels of aggrecan and COL2A1 in the rat chondrocytes, whereas the expression levels of aggrecan and COL2A1 II were increased in chondrocytes co-treated with TNF- $\alpha$ and vitamin D3.

Aggrecanases belong to the ADAMTS family and the most effective aggrecanases associated with joint diseases are ADAMTS-4 (19) and ADAMTS-5 (20). Therefore, the ability of vitamin D3 to regulate the expression levels of ADAMTS-4 and ADAMTS-5 may be of therapeutic value. The current study revealed that the expression levels of ADAMTS-4 and ADAMTS-5 were significantly increased in the rat chondrocytes induced by TNF- $\alpha$, whereas this increase was subsequently inhibited by the co-treatment with vitamin D3.

Collagen degradation mainly occurs through the action of MMPs and increased expression levels of MMPs have been previously closely associated with the progression of OA $(21,22)$. Among the various MMPs, MMP-13 is the main enzyme involved in cartilage erosion in OA because of its proteolytic activity over COL2A1, the main component of cartilage ECM $(23,24)$. Therefore, inhibiting the expression levels of MMP-13 may lead to cartilage protection and therefore have therapeutic value in the treatment of OA $(25,26)$. MMP-3 has also been discovered to be closely associated with the progression of OA and increased MMP-3 expression levels have been observed to promote collagen degradation in the cartilage matrix (27). In a previous study, the abnormal expression levels of MMP-3 were related to the pathogenesis of OA (28). Thus, the enzymes released by chondrocytes in response to certain stimuli are closely associated with the progression of cartilage degradation in OA. The results of the present study demonstrated that the expression levels of
MMP-3 and MMP-13 in chondrocytes stimulated by TNF- $\alpha$ were significantly reduced following co-treatment with vitamin D3 compared with the chondrocytes treated with TNF- $\alpha$ alone. Therefore, it was hypothesized that vitamin D3 served a protective role in the cartilage by blocking the expression and activation of MMPs and ADAMTs, thus blocking the development and progression of OA.

The Wnt/ $\beta$-catenin signaling pathway is an important pathway involved in numerous biological processes, including cell proliferation, migration and differentiation, cartilage homeostasis (29) and joint remodeling (30). In a previous study, the inhibition of the Wnt/ $\beta$-catenin signaling pathway protected against cartilage degradation in OA (31). The upregulation of $\beta$-catenin in degraded cartilage indicated that the stimulation of the Wnt signaling pathway led to cartilage loss (32). In addition, in animal models of OA, $\beta$-catenin was found to be overexpressed in articular cartilage (33). In the present study, TNF- $\alpha$ treatment increased the expression levels of Wnt-3a and nuclear $\beta$-catenin in the chondrocytes. Notably, the Wnt/ $\beta$-catenin pathway inhibitor PNU-74654 inhibited the expression levels of Wnt-3a and nuclear $\beta$-catenin, inhibited the TNF- $\alpha$-induced decreases in ACAN and COL2A expression levels and TNF- $\alpha$-induced increases in the expression levels of MMPs and ADAMTs in chondrocytes. These findings indicated that TNF- $\alpha$ may activate the Wnt $/ \beta$-catenin signaling pathway in chondrocytes. The co-treatment with vitamin D3 also inhibited the TNF- $\alpha$-induced expression levels of Wnt- $3 \mathrm{a}$ and nuclear $\beta$-catenin, the TNF- $\alpha$-induced reduced expression levels of ACAN and COL2A1, and the TNF- $\alpha$-induced increased expression levels of the MMPs and ADAMTs in chondrocytes. These results suggested that the protective effect of vitamin D3 on chondrocytes may be at least partially mediated through the inhibition of the Wnt/ $\beta$-catenin signaling pathway. Other signaling pathways are also thought to be involved in the regulation of MMPs and ADAMTs in chondrocytes, thus further studies are required to determine the mechanisms by which vitamin D3 regulates MMPs and ADAMTs.

In conclusion, the results of the present study provided evidence to suggest that vitamin D3 may inhibit the expression levels of MMP-3, MMP-13, ADAMTS-4 and ADAMTS-5 through inhibiting the $\mathrm{Wnt} / \beta$-catenin signaling pathway. These data suggested that vitamin D3 may have therapeutic value in the prevention and treatment of OA. 


\section{Acknowledgements}

Not applicable.

\section{Funding}

The present study was supported by the Foundation of Tianjin Union Medical Centre (grant no. 2017YJ013).

\section{Availability of data and materials}

The datasets used and/or analyzed during the current study are available from the corresponding author on reasonable request.

\section{Authors' contributions}

MT designed the experiments. TY and WS performed the experiments. YD, YS, YR and WH analyzed data. All authors contributed to the writing of the manuscript. All authors read and approved the final manuscript.

\section{Ethics approval and consent to participate}

The present study was approved by the Animal Care and Use Committee of Tianjin Union Medical Center and Affiliated Zhongshan Hospital of Dalian University.

\section{Patient consent for publication}

Not applicable.

\section{Competing interests}

The authors declare that they have no competing interests.

\section{References}

1. Bortoluzzi A, Furini $F$ and Scirè $C A$ : Osteoarthritis and its management - Epidemiology, nutritional aspects and environmental factors. Autoimmun Rev 17: 1097-1104, 2018.

2. Kanemitsu M, Nakasa T, Shirakawa Y, Ishikawa M, Miyaki S and Adachi N: Role of vasoactive intestinal peptide in the progression of osteoarthritis through bone sclerosis and angiogenesis i n subchondral bone. J Orthop Sci: Jan 9, 2020 (Epub ahead of print).

3. Majeed MH, Sherazi SAA, Bacon D and Bajwa ZH: Pharmacological treatment of pain in osteoarthritis: A descriptive review. Curr Rheumatol Rep 20: 88, 2018.

4. Cao Y, Winzenberg T, Nguo K, Lin J, Jones G and Ding C: Association between serum levels of 25-hydroxyvitamin $\mathrm{D}$ and osteoarthritis: A systematic review. Rheumatology (Oxford) 52: $1323-1334,2013$

5. Tetlow LC and Woolley DE: Expression of vitamin D receptors and matrix metalloproteinases in osteoarthritic cartilage and human articular chondrocytes in vitro. Osteoarthritis Cartilage 9: 423-431, 2001

6. Sun X, Huang H, Pan X, Li S, Xie Z, Ma Y, Hu B, Wang J, Chen $Z$ and Shi P: EGR1 promotes the cartilage degeneration and hypertrophy by activating the Krüppel-like factor 5 and $\beta$-catenin signaling. Biochim Biophys Acta Mol Basis Dis 1865: 2490-2503, 2019.

7. De Santis M, Di Matteo B, Chisari E, Cincinelli G, Angele P, Lattermann C, Filardo G, Vitale ND, Selmi C and Kon E: The role of Wnt pathway in the pathogenesis of OA and its potential therapeutic implications in the field of regenerative medicine. BioMed Res Int 2018: 7402947, 2018.
8. Gröschel C, Aggarwal A, Tennakoon S, Höbaus J, Prinz-Wohlgenannt M, Marian B, Heffeter P, Berger W and Kállay E: Effect of 1,25-dihydroxyvitamin D3 on the Wnt pathway in non-malignant colonic cells. J Steroid Biochem Mol Biol 155 (Pt B): 224-230, 2016.

9. Nusse R and Clevers H: Wnt/ $\beta$-Catenin Signaling, Disease, and Emerging Therapeutic Modalities. Cell 169: 985-999, 2017.

10. Zhou B, Chen D, Xu H and Zhang X: Proliferation of rabbit chondrocyte and inhibition of IL-1 $\beta$-induced apoptosis through MEK/ERK signaling by statins. In Vitro Cell Dev Biol Anim 53: 124-131, 2017.

11. Livak KJ and Schmittgen TD: Analysis of relative gene expression data using real-time quantitative PCR and the $2(-\Delta \Delta$ C(T)) method. Methods 25: 402-408, 2001.

12. McCarty EC: Articular Cartilage: The Search for the Holy Grail of Treatment and Restoration. Clin Sports Med 36: xv-xvi, 2017.

13. Castillo EC, Hernandez-Cueto MA, Vega-Lopez MA, Lavalle C, Kouri JB and Ortiz-Navarrete V: Effects of vitamin D supplementation during the induction and progression of osteoarthritis in a rat model. Evid Based Complement Alternat Med 2012: $156563,2012$.

14. Hunt MA, Charlton JM and Esculier JF: Osteoarthritis year in review 2019: Mechanics. Osteoarthritis Cartilage 28: 267-274, 2020.

15. Zeng YF, Wang R, Bian Y, Chen WS and Peng L: Catalpol attenuates IL-1 $\beta$ induced matrix catabolism, apoptosis and inflammation in rat chondrocytes and inhibits cartilage degeneration. Med Sci Monit 25: 6649-6659, 2019.

16. Zhao Y, Li Y, Qu R, Chen X, Wang W, Qiu C, Liu B, Pan X, Liu L, Vasilev K, et al: Cortistatin binds to TNF- $\alpha$ receptors and protects against osteoarthritis. EBioMedicine 41: 556-570, 2019.

17. Urban $\mathrm{H}$ and Little $\mathrm{CB}$ : The role of fat and inflammation in the pathogenesis and management of osteoarthritis. Rheumatology (Oxford) 57 (Suppl 4): iv10-iv21, 2018.

18. Tian J, Rui YJ, Xu YJ and Zhang SA; J. T: miR-143-3p regulates early cartilage differentiation of BMSCs and promotes cartilage damage repair through targeting BMPR2. Eur Rev Med Pharmacol Sci 22: 8814-8821, 2018.

19. Chijiiwa M, Mochizuki S, Kimura T, Abe H, Tanaka Y, Fujii Y, Shimizu H, Enomoto H, Toyama Y and Okada Y: CCN1 (Cyr61) is overexpressed in human osteoarthritic cartilage and inhibits ADAMTS-4 (aggrecanase 1) activity. Arthritis Rheumatol 67: $1557-1567,2015$.

20. Rogerson FM, Last K, Golub SB, Gauci SJ, Stanton H, Bell KM and Fosang AJ: ADAMTS-9 in Mouse Cartilage Has Aggrecanase Activity That Is Distinct from ADAMTS-4 and ADAMTS-5. Int J Mol Sci 20: 573, 2019.

21. Seidl CI and Murphy CL; CI: Dual and Opposing Regulation of MMP1 and MMP13 by Both Arms of miR-675 in Human Articular Chondrocytes. Cell Physiol Biochem 53: 172-185, 2019.

22. Malemud CJ: Inhibition of MMPs and ADAM/ADAMTS. Biochem Pharmacol 165: 33-40, 2019.

23. Yu HT, Gu CZ and Chen JQ: MiR-9 facilitates cartilage regeneration of osteoarthritis in rabbits through regulating Notch signaling pathway. Eur Rev Med Pharmacol Sci 23: 5051-5058, 2019.

24. Mazur CM, Woo JJ, Yee CS, Fields AJ, Acevedo C, Bailey KN, Kaya S, Fowler TW, Lotz JC, Dang A, et al: Osteocyte dysfunction promotes osteoarthritis through MMP13-dependent suppression of subchondral bone homeostasis. Bone Res 7: 34, 2019.

25. Shu CC, Flannery CR, Little CB and Melrose J: Catabolism of fibromodulin in developmental rudiment and pathologic articular cartilage demonstrates novel roles for MMP-13 and ADAMTS-4 in C-terminal processing of SLRPs. Int J Mol Sci 20: $579,2019$.

26. Tang LP, Ding JB, Liu ZH and Zhou GJ; LP: LncRNA TUG1 promotes osteoarthritis-induced degradation of chondrocyte extracellular matrix via miR-195/MMP-13 axis. Eur Rev Med Pharmacol Sci 22: 8574-8581, 2018.

27. van Geffen EW, van Caam APM, Schreurs W, van de Loo FA, van Lent PLEM, Koenders MI, Thudium CS, Bay-Jensen AC, Blaney Davidson EN and van der Kraan PM: IL-37 diminishes proteoglycan loss in human OA cartilage: Donor-specific link between IL-37 and MMP-3. Osteoarthritis Cartilage 27: 148-157, 2019.

28. Guo L, Hao R, Tian F, An N and Wang K: Interferon regulatory factor 5 (IRF5) regulates the expression of matrix metalloproteinase-3 (MMP-3) in human chondrocytes. Int Immunopharmacol 55: 231-236, 2018. 
29. Miyamoto K, Ohkawara B, Ito M, Masuda A, Hirakawa A, Sakai T, Hiraiwa $\mathrm{H}$, Hamada $\mathrm{T}$, Ishiguro $\mathrm{N}$ and Ohno $\mathrm{K}$ : Fluoxetine ameliorates cartilage degradation in osteoarthritis by inhibiting Wnt/ $\beta$-catenin signaling. PLoS One 12: e0184388, 2017.

30. Ma L, Wu J and Jin QH: The association between parathyroid hormone 1-34 and the Wnt/ $\beta$-catenin signaling pathway in a rat model of osteoarthritis. Mol Med Rep 16: 8799-8807, 2017.

31. Sun Y, Wang F, Sun X, Wang X, Zhang L and Li Y: CX3CR1 regulates osteoarthrosis chondrocyte proliferation and apoptosis via Wnt $/ \beta$-catenin signaling. Biomed Pharmacother 96: 1317-1323, 2017.
32. Yuasa T, Otani T, Koike T, Iwamoto $M$ and Enomoto-Iwamoto $M$ : Wnt/beta-catenin signaling stimulates matrix catabolic genes and activity in articular chondrocytes: Its possible role in joint degeneration. Lab Invest 88: 264-274, 2008.

33. Li WJ, Tang LP, Xiong Y, Chen WP, Zhou XD, Ding QH and Wu LD: A possible mechanism in DHEA-mediated protection against osteoarthritis. Steroids 89: 20-26, 2014. 